\title{
Numerical Simulation of Self-Sensing Time Domain Reflectometry for Damage Detection of Carbon Fiber Reinforced Polymer Plate*
}

\author{
Akira TODOROKI**, Hiroumi KUROKAWA*** and Yoshihiro MIZUTANI** \\ ** Department of Mechanical Sciences and Engineering, \\ Tokyo Institute of Technology, \\ 2-12-1 Ookayama, Meguro-ku, Tokyo, 1528552, Japan \\ E-mail: atodorok@ginza.mes.titech.ac.jp \\ *** Graduate Student, Tokyo Institute of Technology, \\ 2-12-1 Ookayama, Meguro-ku, Tokyo, 1528552, Japan
}

\begin{abstract}
This study is an analytical investigation of time domain reflectometry (TDR) for carbon fiber reinforced polymer (CFRP) composites using numerical simulation software. This process is called self-sensing TDR because the carbon fibers themselves are used as the sensors. A previous study conducted experimental investigations of this process using parallel aluminum plates. This study uses finite difference time domain analysis for simulations of a transmission line structure for the self-sensing TDR. Details of the effects of the orthotropic electric conductance are investigated analytically. The simulation results show that the electromagnetic waves propagate rapidly in the transverse direction and that the effects of the orthotropic conductance seem to be negligible. An additional simulation using a pair of square CFRP plates reveals that the electromagnetic wave is affected by the orthotropic conductance. The effect is nevertheless very small when compared with the actual ratio of the electric conductance between the fiber direction and the transverse direction.
\end{abstract}

Key words: Carbon Fibers, Polymer Matrix Composites, Time Domain Reflectometry, Smart Materials, Electrical Conductance, Fiber Breakage

\section{Introduction}

Laminated carbon fiber reinforced polymer (CFRP) composites are widely used in the primary structures of aircraft structural components. Laminated CFRP structures have lower strength in interlaminar areas, rendering them vulnerable to damage from low-velocity impact loads. These characteristics underscore the need for a CFRP structural damage monitoring system. The reinforcing carbon fibers have high electrical conductivity. Therefore, they are potentially useful as sensors for damage detection in the CFRP structures. Using the structural carbon fibers as sensors, we have created a self-sensing $\operatorname{method}^{1)-8)}$.

Time domain reflectometry (TDR) measures the time difference between the reflected pulse signals from the end of a transmission line and those from the damage. It detects the location of the damage point in the target transmission line from the measured time difference. A pulse generator is used to send a pulse signal to the target transmission line. The reflected pulse signals are then measured using a digital oscilloscope. The TDR method has already been applied to structural damage monitoring ${ }^{9)-12)}$. In a previous study, the

*Received 18 Sep., 2012 (No. 12-0380) [DOI: 10.1299/jmmp.6.1062]

Copyright $\odot 2012$ by JSME 
authors proposed self-sensing TDR for a CFRP plate. The CFRP plate was used to form a transmission line. The damage of the CFRP was detected from the reflected pulse signal ${ }^{13}$. The experimentally obtained results from that earlier study confirmed the applicability of the self-sensing TDR method for damage detection in a CFRP plate. The experimentally obtained results from the multiple electrodes, however, did not provide the location in the transverse direction. Information about the location in the transverse direction was lost in the experiments. In this study, analyses were performed to investigate the effects of the anisotropic electric conductance of the CFRP plates for self-sensing TDR. For these analyses, the finite difference time domain (FDTD) method was used to analyze the electric field of the transmission line used for self-sensing TDR. To remove the specimen width effect, a square CFRP plate specimen was selected for an additional simulation. The simulation using the square CFRP plate reveals the anisotropic conductance effect.

\section{Principle of Self-Sensing TDR}

The TDR method uses a pulse signal in a transmission line. The pulse signal reflected from the transmission line is measured. The results are observed in a figure in which the abscissa is time and the ordinate is the voltage. The TDR method requires a wave generator, an oscilloscope, and a target cable, as shown in Fig. 1. The wave generator produces a pulse wave signal, which is sent into the directional coupler. The signal propagates only into the target cable through the directional coupler. Part of the signal is reflected at the input end of the cable because of a slight difference in the characteristic impedance. The remainder of the signal propagates in the target cable. The signal input into the target cable is divided into reflected and transmitted signals at the damage point. The reflected signal returns and is measured at the oscilloscope. The time difference between the input signal and the reflected signal indicates the distance to the damage point after multiplication by the signal speed. Using the TDR method, the damage and its location are thus measurable. The distance $L$ from the input end to the damage point is calculated using the following equation ${ }^{14)}$.

$$
L=\frac{V_{p} \Delta T}{2}
$$

In the equation, $V_{\mathrm{p}}$ is the transmission velocity, and $\Delta T$ denotes the time difference between the input signal and the reflected signal. The transmission velocity $V_{\mathrm{p}}$, which is affected by the transmission line, is slightly lower (approximately 0.6-0.9) than the velocity of light. For this study, the cable is replaced with the CFRP plate.

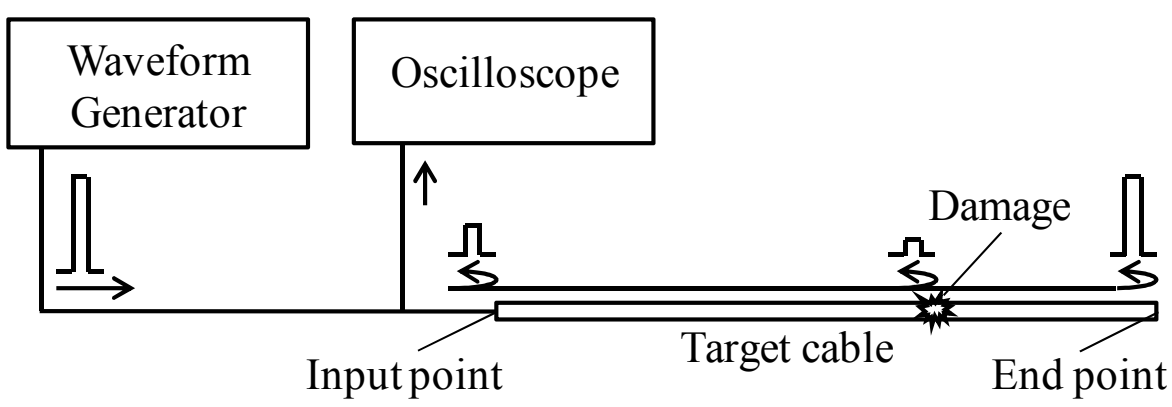

Fig. 1. Schema of TDR measurement.

When the cable is simply replaced with the CFRP plate, the characteristic impedance of the CFRP plate differs greatly from that of the coaxial cable that is used to connect the wave generator and the oscilloscope. This arrangement causes perfect reflection to occur at the input end of the CFRP plate, and thus the pulse signal does not propagate in the CFRP plate. 
An impedance-matching process is therefore indispensable for the TDR method of the CFRP plate. Our previous study performed impedance matching using a parallel aluminum plate. Figure 2 presents the experimental setup for the self-sensing TDR used in that study 13) . A prepreg (Pyrofile MR380; Mitsubishi Rayon Co., Ltd.) was used, from which a CFRP plate was fabricated with a stacking sequence of $\left[0_{4}\right]_{\mathrm{T}}$. The $1980-\mathrm{mm}$-long CFRP plate was $120 \mathrm{~mm}$ wide. The specimen was sandwiched with an aluminum plate. Glass fiber heat insulating materials were used to keep the specimen at $130^{\circ} \mathrm{C}$ for $2 \mathrm{hr}$.

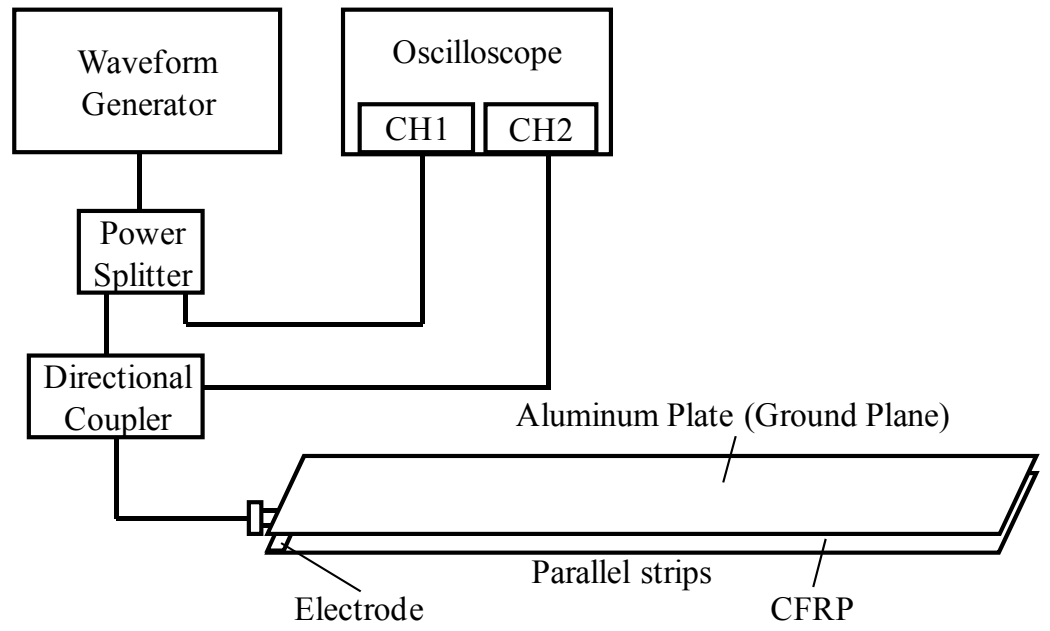

Fig. 2. Schema of self-sensing TDR for CFRP.

\section{Numerical Simulation}

The frequency of the pulse signal was approximately $200 \mathrm{MHz}$ in the previous paper, meaning that the wavelength is approximately $1.5 \mathrm{~m}$. When the conductive line is longer than $1 / 100$ of the wavelength, it must then be regarded as a transmission line that comprises two conductive materials. In this study, the FDTD method is adopted for the analysis. The method solves Maxwell's equations directly using the finite difference method. Maxwell's equations are as follows.

$$
\begin{aligned}
& \frac{d \mathbf{D}}{d t}=-\nabla \times \mathbf{H}-\mathbf{J} \\
& \frac{d \mathbf{B}}{d t}=-\nabla \times \mathbf{E} \\
& \mathbf{D}=\mathbf{\varepsilon} \mathbf{E} \\
& \mathbf{B}=\boldsymbol{\mu} \mathbf{H}
\end{aligned}
$$

Therein, D stands for the electrical flux density vector, B represents the magnetic flux density vector, $\mathbf{E}$ signifies the electrical field strength vector, $\mathbf{H}$ is the magnetic field strength vector, $\varepsilon$ is the dielectric constant, and $\mu$ denotes the magnetic permeability. $\mathbf{J}$ is the electric current density vector. When the calculation domain is inside a conductive material, $\mathbf{J}$ is written as

$$
\mathbf{J}=\boldsymbol{\sigma} \mathbf{E}
$$

where $\sigma$ denotes the electric conductance. The quantities $\varepsilon, \mu$, and $\sigma$ are $3 \times 3$ matrix values for anisotropic materials. When the calculation domain comprises an isotropic material, $\varepsilon, \mu$ and $\sigma$ then become diagonal matrixes with identical elements.

To solve Maxwell's equations, the free "MEEP" FDTD simulation software is used ${ }^{15)}$. An analytical model is presented in Fig. 3. The 1980-mm-long CFRP plate is $120 \mathrm{~mm}$ wide. The spacing between the aluminum plate and the CFRP plate is $10 \mathrm{~mm}$. As a boundary 
condition, a surrounding box area is set to match the layer perfectly (perfectly absorbed layer). The spacing between the specimen and the layer is $10 \mathrm{~mm}$. For the FDTD method, a regular hexahedron mesh division is generally used. The length of each side of the hexahedron mesh is usually set to less than one-tenth of the wavelength. In this study, the length is set at $1 \mathrm{~mm}$, meaning that the maximum available frequency for the FDTD analysis using this mesh is $30 \mathrm{GHz}$. For the time step of the analysis, the FDTD requires Courant's stability condition.

$$
\Delta t \leq \frac{1}{c \sqrt{\left(\frac{1}{\Delta x}\right)^{2}+\left(\frac{1}{\Delta y}\right)^{2}+\left(\frac{1}{\Delta z}\right)^{2}}}
$$

Therein, $\mathrm{c}$ is the speed of light. Because the length of each side of the hexahedron mesh is 1 $\mathrm{mm}(\Delta x=\Delta y=\Delta z=1)$, the time step $\Delta t$ is set to $1.67 \times 10^{-3} \mathrm{~ns}$.

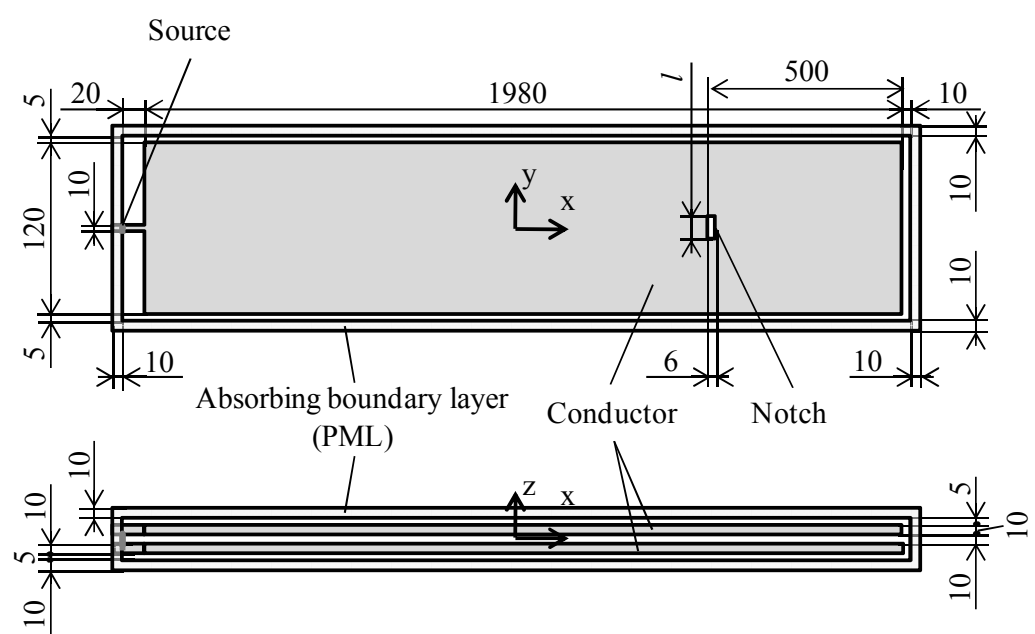

Fig. 3. Computational model of TDR of CFRP for the FDTD method.

As the dimensions of the microstructure of CFRP from the perspective of an electrical circuit are approximately 10-50 $\mu \mathrm{m}$ (the spacing between the fiber contact points), the reference dimension of the microstructure is sufficiently small when compared with the hexahedron mesh length, which indicates that the CFRP can be treated as a homogeneous orthotropic material. Because the carbon fibers and the epoxy resin are not magnetic materials, the relative magnetic permeability of the CFRP is set to 1 . The other electrical properties required for the FDTD analysis are shown in Table 1. For the analyses, two types of CFRP are prepared to compare the effects of the orthotropic conductance: Pyrofile \#380 and IM600/133. The Pyrofile \#380 is a normal CFRP but IM600/133 has strongly orthotropic conductance. In this study, analyses of four types of line are performed.

(1) A transmission line with an aluminum strip and a CFRP strip made from Pyrofile \#380.

(2) A transmission line with an aluminum strip and a CFRP strip made from IM600/133.

(3) A transmission line with two CFRP strips made from IM600/133.

(4) A transmission line with two CFRP square plates made from IM600/133.

Pulse signals of two types are prepared here: signals of a similar frequency to those used in the experiments (see Fig. 4(a), $0.15 \mathrm{GHz}$ ) and signals of a higher frequency than those used in the experiments (see Fig. 4(b), 2 GHz). The higher frequency pulse signal is used to clarify the effects of the orthotropic conductance of CFRP because the rapid increase of the pulse signal clarifies the difference. For the FDTD analyses, the pulse signal is input at the middle of the left end of the specimen. The pulse signal used here is a sine 
wave, and it is different from the single of a half-sine-wave pulse used in the previous experiments ${ }^{(13)}$. As the frequency of the input signal is very high, most of the input signal propagates in the transmission line as electro-magnetic waves here.

Table 1. Electrical conductance used for the FDTD method.

\begin{tabular}{cccc}
\hline & $\begin{array}{c}\text { Aluminum } \\
{\left[(\Omega m)^{-1}\right]}\end{array}$ & $\begin{array}{c}\text { Pyrofile\#380 } \\
{\left[(\Omega m)^{-1}\right]}\end{array}$ & $\begin{array}{c}\text { IM600/133 } \\
{\left[(\Omega m)^{-1}\right]}\end{array}$ \\
\hline Longitudinal & $3.69 \times 10^{7}$ & $4.10 \times 10^{3}$ & $3.46 \times 10^{4}$ \\
Transverse & $3.69 \times 10^{7}$ & 3.30 & 1.22 \\
Thickness & $3.69 \times 10^{7}$ & 3.30 & $3.24 \times 10^{3}$ \\
\hline
\end{tabular}

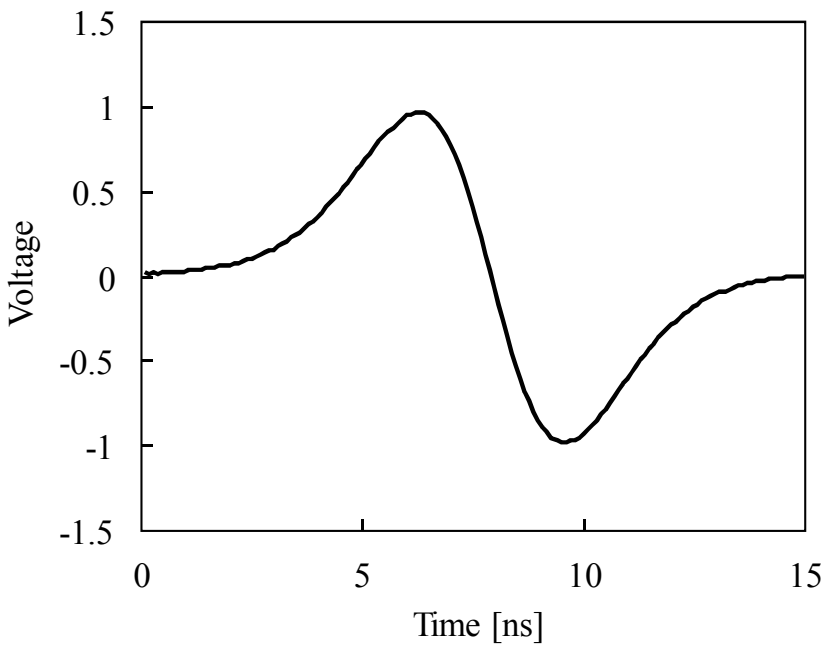

(a) Approximately $0.15[\mathrm{GHz}]$.

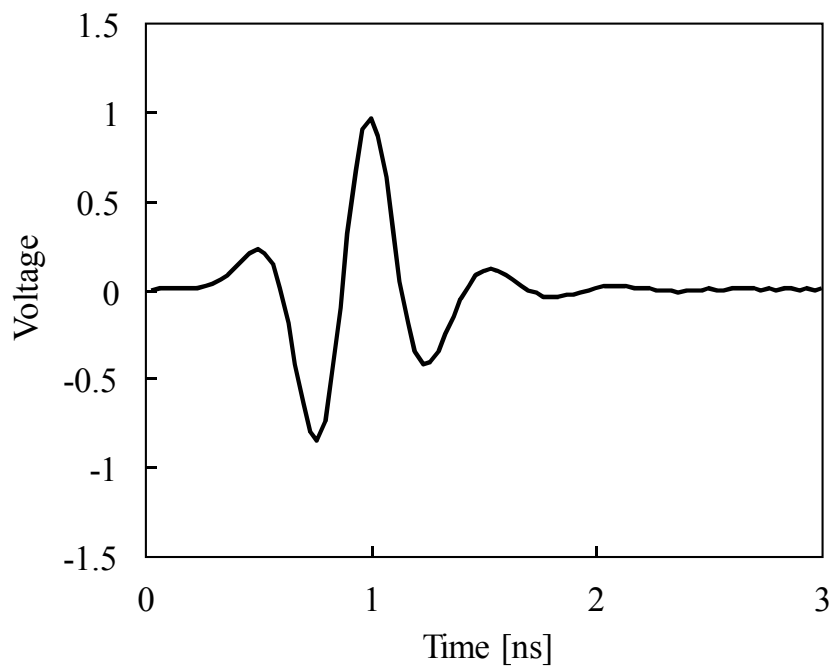

(b) Approximately $2.0[\mathrm{GHz}]$.

Fig. 4. Input signals for the FDTD simulations. 


\section{Results and Discussion}

Figure 5 presents the results for the reflected pulse signal from the end of a specimen of a transmission line composed of an aluminum plate and a CFRP plate of Pyrofile \#380. The abscissa shows the time difference from the peak of the input signal. The ordinate is the measured voltage. The solid curve is the reflected signal calculated in the FDTD analysis, and the dashed curve is the experimentally obtained reflected pulse signal. In the experiments of the previous paper, a single pulse signal was input to a strip type transmission line ${ }^{13)}$. For the FDTD analysis, a sine wave is used because of the lack of information about an appropriate wave shape (single half-sine wave is used in the previous experiments ${ }^{(13)}$ ). For this reason, the negative signal is observed only for the FDTD analysis results.

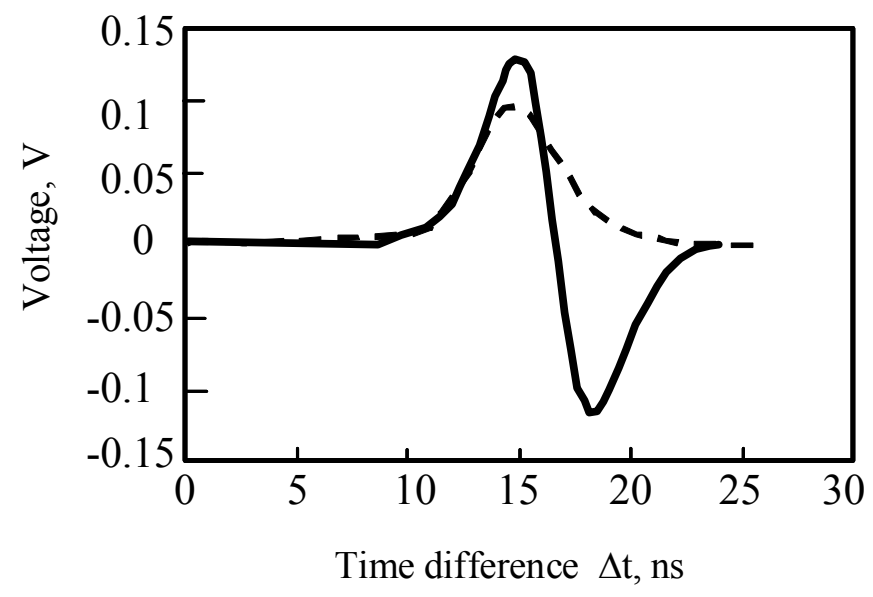

Fig. 5. Reflected pulse signal obtained from the FDTD method (solid curve denotes FDTD; dashed curve denotes experiment).

The measured velocity of the FDTD signal is $2.64 \times 10^{8} \mathrm{~m} / \mathrm{s}$. The measured velocity of the signal from the experiments is $2.66 \times 10^{8} \mathrm{~m} / \mathrm{s}$. The difference between the two is negligible, and the reflected wave height of the simulation result resembles that of the experimentally obtained results. These results show that the FDTD analysis yields excellent agreement with the experimentally obtained results for the transmission line made from the aluminum plate and the CFRP plate. Because the input signal of $0.15 \mathrm{GHz}$ has a wider wavelength, the $2 \mathrm{GHz}$ signal is used for the remainder of the analyses to clarify the signal wave propagation behavior in detail.

Figure 6 shows the propagation of the electric field of the $z$-direction $\left(\mathrm{E}_{\mathrm{z}}\right)$ to the $x$-direction. The strength of $\mathrm{E}_{\mathrm{z}}$ is shown using a color contour plot. The initial electric field is applied at the middle point of the left end of the specimen. The electric field propagates immediately to the end of specimen width (y-direction), and $\mathrm{E}_{\mathrm{z}}$ becomes a uniform wave in the transverse direction soon after the propagation in the $x$-direction (see results after $1.0 \mathrm{~ns}$ and $3.0 \mathrm{~ns}$ ). After propagation of approximately $200 \mathrm{~mm}$ in the $x$-direction, $\mathrm{E}_{\mathrm{z}}$ becomes uniform in the $y$-direction for the normal CFRP (Pyrofile \#380).

Figure 7 shows the propagation of the electric field of $\mathrm{E}_{\mathrm{z}}$ in the $x$-direction of the strip specimen with damage. At the damage location, the pulse signal becomes completely uniform in the $y$-direction, and the reflected pulse wave is also a uniform plane wave in the $y$-direction. For this reason, the multiple electrode methods used in the previous experimental study detected damage equally from every electrode formed at the left end of the specimen ${ }^{13}$. This simulation result shows that the effect of the orthotropic conductance of the normal CFRP is negligible for the strip-type specimen for self-sensing TDR. 


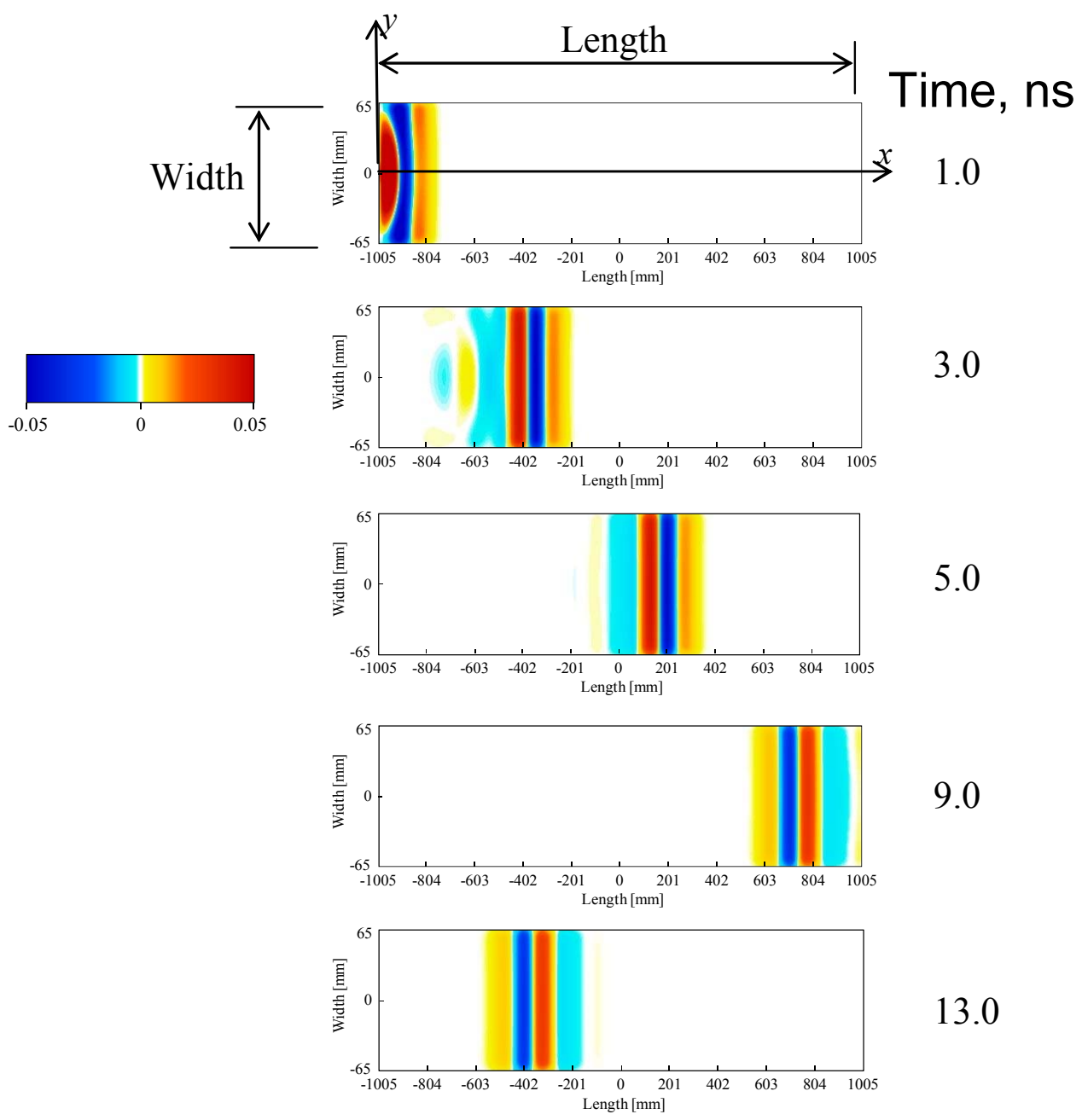

Fig. 6. Electrical field of $\mathrm{E}_{\mathrm{z}}$ at $z=0$ of Pyrofile $\# 380$ without damage.

Figure 8 shows the propagation of the electric field $\mathrm{E}_{\mathrm{z}}$ in the $x$-direction of the strip type specimen of the IM600/133 composites. As Table 1 shows, IM600/133 has strong orthotropic electric conductance. The result, however, shows no difference to that obtained with the normal CFRP.

Figure 9 shows the propagation of the electric field $\mathrm{E}_{\mathrm{z}}$ in the $x$-direction of the strip type specimen of the IM600/133 composites. In this simulation, a pair of IM600/133 plates was selected to make a transmission line rather than using an aluminum plate. The results show that $\mathrm{E}_{\mathrm{z}}$ at $1 \mathrm{~ns}$ exhibits a slow propagation rate in the $y$-direction. The effect of the strong orthotropic conductance, however, becomes negligible at 3 ns. The results shown in Fig. 8 and Fig. 9 show that the effect of the strong orthotropic conductance is very small for the self-sensing TDR of the strip type specimen. This small effect might originate from the small dimensions of the specimen width in the $y$-direction.

To confirm the effect of the specimen width of the strip type specimen, an additional simulation was performed. Two IM600/133 square plates were used to produce a transmission line. The square plate length is $1000 \mathrm{~mm}$, and the other dimensions are the same as those in Fig. 3. The result after $4 \mathrm{~ns}$ is presented in Fig. 10. As shown in Fig. 10, $\mathrm{E}_{\mathrm{z}}$ is long in the $x$-direction and is a thin ellipse. The aspect ratio of the ellipse is, however, 
approximately equal to 5 . The ratio of the electric conductance in the fiber direction to that in the transverse direction is $2.8 \times 10^{4}$, as shown in Table 1 . The aspect ratio of $\mathrm{E}_{\mathrm{z}}$ is too small when compared with the conductance ratio of the IM600/133 composites. Therefore, the self-sensing TDR is affected by the orthotropic conductance of the CFRP, but the effect is quite small when compared with the actual conductance ratio because $\mathrm{E}_{\mathrm{z}}$ propagates in the dielectric material. The effect might be influenced by the dimensions of the spacing between the two CFRP plates. However, these are subjects for our future work.
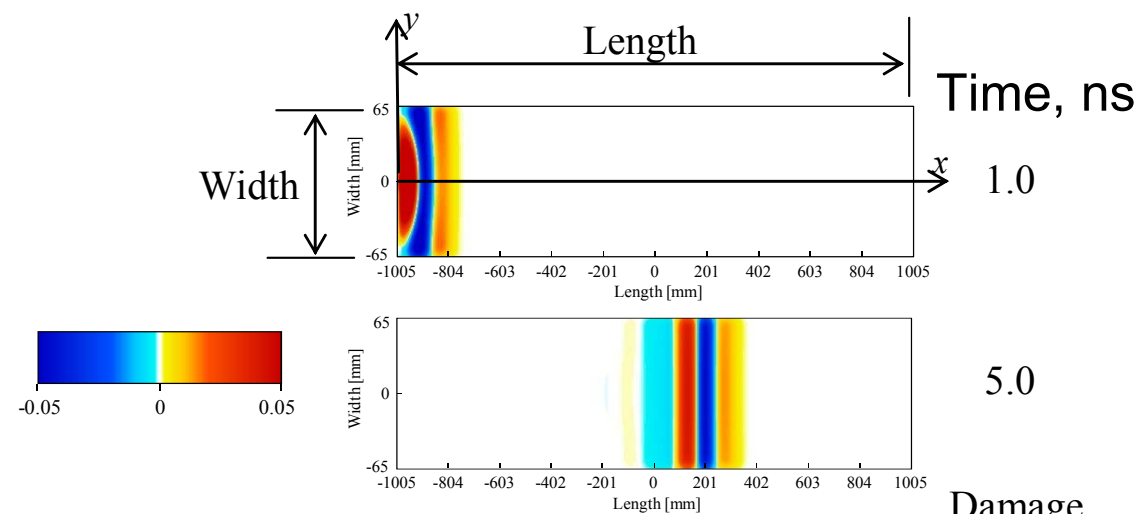

Reflected
signal

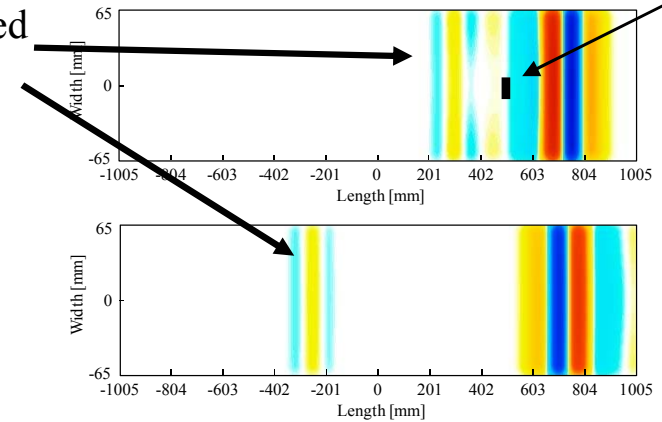

Damage

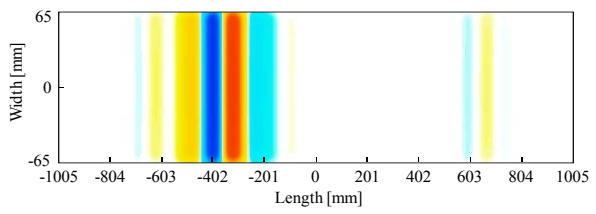

Fig. 7. Electrical field of $E_{z}$ at $z=0$ of Pyrofile $\# 380$ with damage.

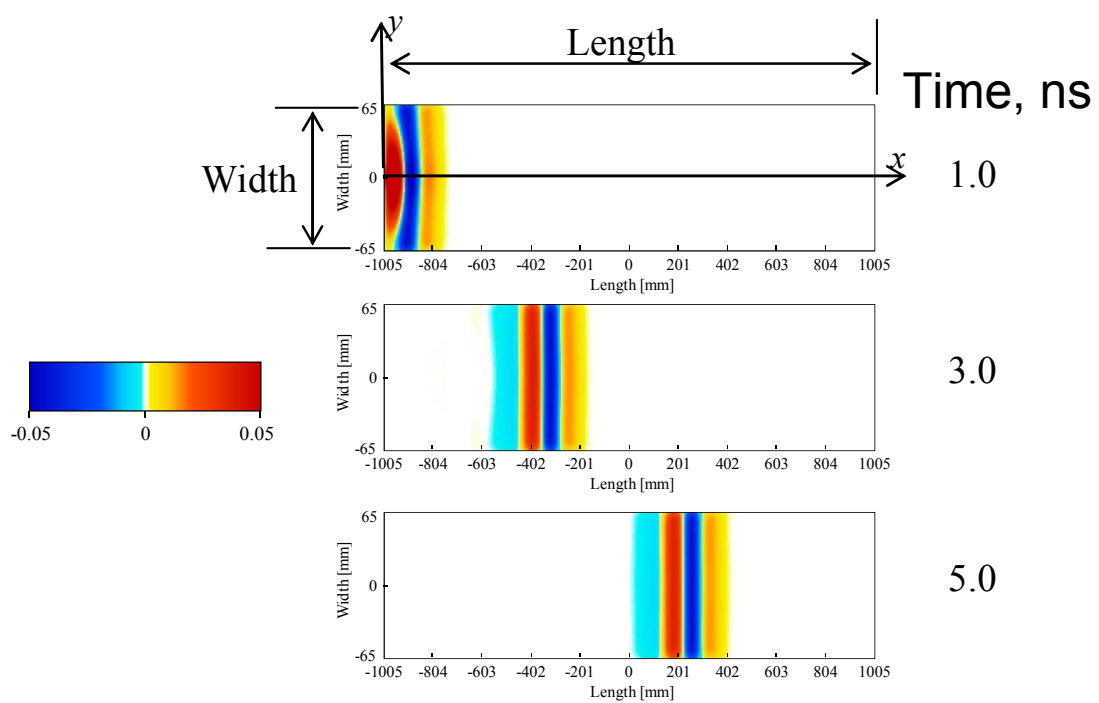

Fig. 8. Electrical field of $E_{z}$ at $z=0$ of IM600/133 without damage. 


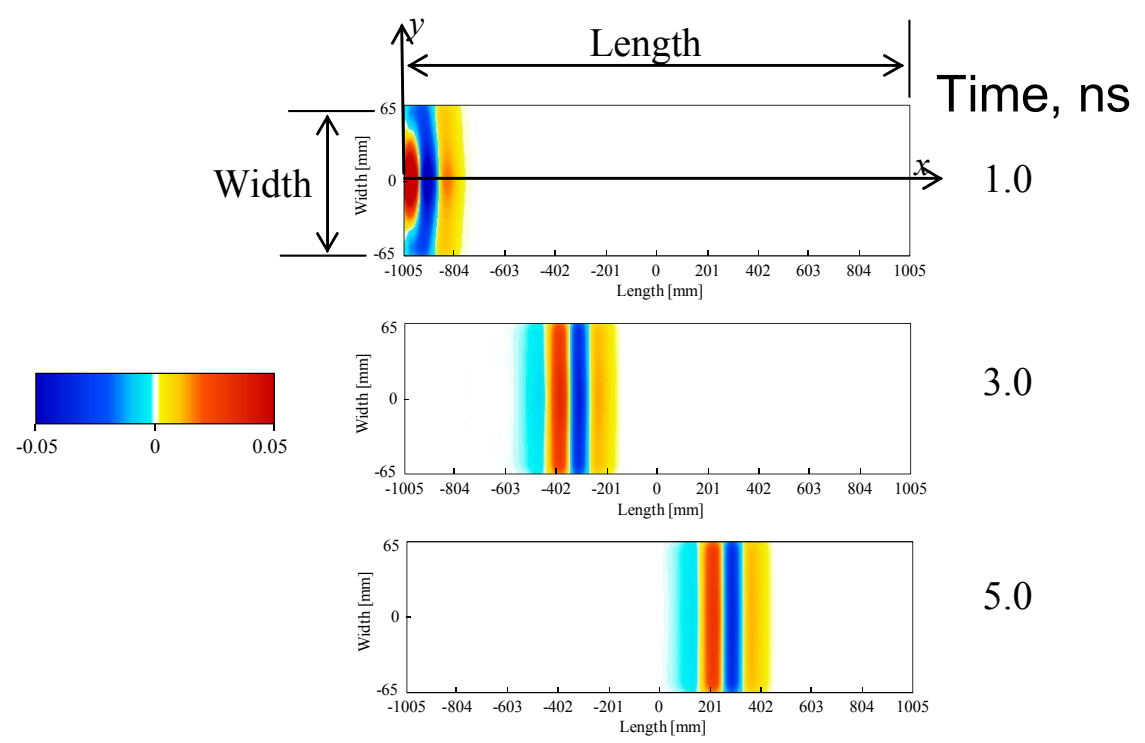

Fig. 9. Electrical field of Ez at $z=0$ of IM600/133 without damage (transmission line using two CFRP plates).

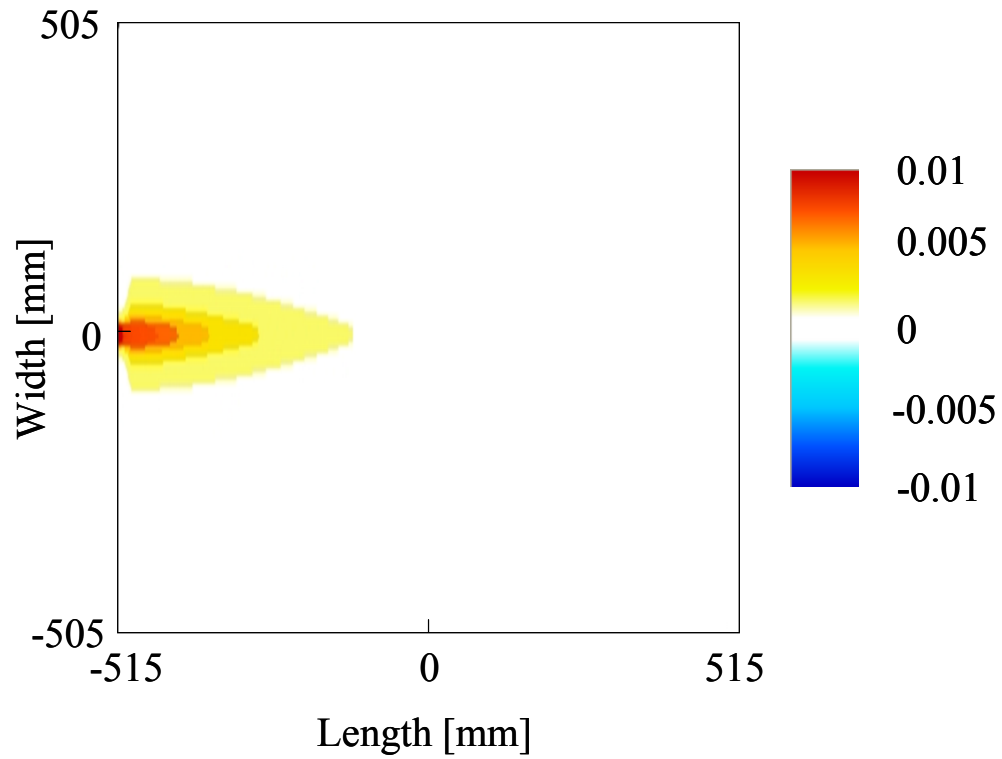

Fig. 10. Electrical field of $\mathrm{E}_{\mathrm{z}}$ at $z=0$ of IM600/133 without damage (square plate transmission line using two CFRP plates at $4 \mathrm{~ns}$ ).

\section{Conclusions}

In this study, self-sensing TDR is specifically addressed and simulations are performed using the FDTD method. The experimentally obtained results from our previous paper demonstrated the applicability of the self-sensing TDR method to damage detection of a CFRP plate. The experimentally obtained results with multiple electrodes, however, did not detect the damage location in the transverse direction. In this study, therefore, analyses were conducted to investigate the effects of the anisotropic electric conductance of CFRP plates on the self-sensing TDR. The results obtained are as follows.

(1) The FDTD simulation result for the no damage scenario is compared with the experimentally obtained results, and the FDTD simulation is shown to provide appropriate results for self-sensing TDR using CFRP plates. 
(2) When using a narrow strip, the transmitted wave propagates in the transverse direction and the effect of the orthotropic conductance is negligible, even for the strongly orthotropic CFRP.

(3) When using a pair of square CFRP plates, the transmitted wave has an orthotropic wave velocity. The ratio of the velocity is, however, smaller than that of the electric conductance.

\section{References}

(1) K. Schulte, Ch. Baron Load and Failure Analyses of CFRP Laminates by Means of Electrical Resistivity Measurements, Comp. Sci. Tech., 1989;Vol.36, No.1, (1989), pp.63-76.

(2) N. Muto, H. Yanagida, T. Nakatsuji, M. Sugita, Y.Ohtsuka, Preventing Fatal Fractures in Carbon-Fibre - Glass-Fibre-Reinforced Plastic Composites by Monitoring Change in Electrical Resistance, J American Ceramic Society,Vol.76, No.4, (1993),pp.875-879.

(3) X. Wang, D.D.L. Chung, Short Carbon Fiber Reinforced Epoxy as a Piezoresistive Strain Sensor. Smart Materials and Structures,Vol.4, No.4, (1995),pp. 363-367.

(4) A. Todoroki, K. Matsuura, H. Kobayashi, Application of Electric Potential Method to Smart Composite Structures for Detecting Delamination, JSME International J, Series A, Vol.38, No.4, (1995), pp.524-530.

(5) P.E. Irving, C.Thiagarajan, Fatigue Damage Characterization in Carbon Fibre Composite Materials using an Electrical Potential Technique, Smart Materials and Structures,Vol.7, No.4, (1998), pp.456-466.

(6) J.C. Abry, Y.K. Choi, A. Chateauminois, B. Dalloz, G. Giraud, M. Salvia, In-situ Monitoring of Damage in CFRP Laminates by using AC and DC measurements, Comp. Sci. Tech., Vol.61, No.6, (2001), pp.855-864.

(7) J.B. Park, T. Okabe, N. Takeda, W.A. Curtin, Electromechanical modeling of unidirectional CFRP composites under tensile loading condition, Composites Part A,Vol.33, No.2, (2002), pp.267-275.

(8) K. Ogi, Y. Takao, Characterization of piezoresistance behavior in a CFRP unidirectional laminate, Comp. Sci. Tech.,Vol.65, No.2, (2005), pp.231-239.

(9) G.D. Chen, S.S. Sun, D. Pommerenke, J.L. Drewniak, G.G. Greene, R.D. McDaniel, A. Belarbi, H.M. Mu, Crack detection of a full-scale reinforced concrete girder with a distributed cable sensor, Smart Materials and Structures,Vol.14,No.3, (2005), pp.S88-S97.

(10) M.W. Lin, J. Thaduri, A.O. Abatan, Development of an electrical time domain Reflectometry (ETDR) distributed strain sensor, Measurement Science and Technology, Vol.16, No.7, (2005), pp.1495-1505.

(11) Y. Okuhara, S.G. Shin, H. Matsubara, H. Yanagida, N. Takeda, Development of conductive FRP containing carbon phase for self-diagnosis structures, Proceedings of SPIE Smart Structures and Materials, Vol.4328, No.1,(2002), pp.314-322.

(12) A. Abu Obaid, S. Yarlagadda, M.K. Yoon, N.E. Hager III, R.C. Domszy, A time-domain reflectometry method for automated measure met of crack propagation in composites during mode I DCB testing, J Comp. Mater.,Vol.40,No.22,(2006), pp.2047-2066.

(13) H. Kurokawa, A. Todoroki, , Y. Mizutani, Damage Monitoring of CFRP plate using Self-sensing TDR method, Journal of Solid Mechanics and Materials Engineering, JSME, paper \#T2-2011-JAR-1110, Vol.6, No.12, (2012).

(14) K.M. O'Connor, C.H. Dowding. "GeoMeasurements by Pulsing TDR Cables and Probes", CRC Press (1999), pp. 15-35.

(15) http://ab-initio.mit.edu/wiki/index.php/Meep 